\title{
Propuesta neurolingüística para potencializar la atención voluntaria en estudiantes Colombianos
}

\author{
Neuro-linguistic proposal to potentiate voluntaryattention in students Colombians
}

\author{
Hilda Velasco Sinisterra ${ }^{1}$ y Judith Yangali Vicente ${ }^{2 a}$ \\ Universidad Privada Norbert Wiener, Perú ${ }^{12}$ \\ ORCID ID: https://orcid.org/0000-0002-9233-05891 \\ ORCID ID : https://orcid.org/0000-0003-0302-5839²
}

Recibido: 15 de junio de 2020

Aceptado: 12 de octubre de 2020

\begin{abstract}
Resumen
El propósito de esta investigación es demostrar la influencia positiva de la propuesta de programación neurolingüística en la mejora potencial de la atención voluntaria de los escolares en instituciones públicas de la zona urbana de Buenaventura, Colombia. El método de trabajo es hipotético deductivo con un nivel de investigación explicativo, el diseño en el estudio fue experimental, de subdiseño cuasi-experimental, corte longitudinal, donde se aplicó un pre-test de evaluación al inicio del ciclo académico y un post-test al final, a dos grupos de estudio divididos como un grupo control y un grupo experimental, la estrategia consistió aplicar la propuesta de programación neurolingüística al grupo experimental para evaluar su efecto y compararlo con el grupo control. Se demostró que existe una influencia positiva de la propuesta de programación neurolingüística ligada a estilos de aprendizajes en la atención voluntaria en estudiantes de transición en Buenaventura, Colombia.
\end{abstract}

Palabras clave: programación neurolingüística, dimensiones preescolar, TDAH atención voluntaria, canales sensoriales, estilos de aprendizaje.

\begin{abstract}
The purpose of the research was to demonstrate the positive influence of the neurolinguistic programming proposal in the potential improvement of the voluntary attention of schoolchildren in public institutions in the urban area of Buenaventura, Colombia. The methodology of this work is hypothetical deductive with an explanatory research level, the design in the study was experimental, with a quasi-experimental subdesign, longitudinal cut, where an evaluation pre-test was applied at the beginning of the academic cycle and a Post-test at the end, to two study groups divided as a control group and an experimental group, the strategy consisted of applying the neurolinguistic programming proposal to the experimental group to evaluate its effect and compare it
\end{abstract}

${ }^{\mathrm{a}}$ Correspondencia al autor:

E-mail: judith.yangali@uwiener.edu.pe 
with the control group. It was shown that there is a positive influence of the neurolinguistic programming proposal linked to learning styles in voluntary attention in transitional students in Buenaventura, Colombia.

Keywords: neurolinguistic programming, preschool dimensions, ADHD voluntary attention, sensory channels, learning styles.

\section{Introducción}

Teniendo en cuenta los diferentes planteamientos frente a las dificultades del aprendizaje que presentan los discentes en el ámbito escolar, es importante tener como referente el contexto internacional, en el cual se ha evidenciado un flagelo imperante a nivel educativo: el TDAH (trastorno de déficit de atención e hiperactividad) ya que a nivel mundial existen serios problemas en el desarrollo evolutivo, pedagógico y personal de los niños y niñas, como son: el bajo rendimiento académico, dificultades para adaptarse a la vida social y serios desajustes emocionales, como lo da a conocer la Fundación INECO (2019), quien aduce que la Organización Mundial de la Salud más del 4\% de la población mundial tiene TDAH, aspecto que genera a nivel escolar, una alarma y un riesgo, debido a que muchos maestros y maestras, no cuentan con la capacitación y especialidad para tratar dichos casos. Abriendo mucho más el panorama del docente en el manejo pedagógico, donde el quehacer del maestro se encuentra bombardeado y saturado por una crisis en el campo escolar desde los procesos inclusivos, las NEE (necesidades educativas especiales) y el desconocimiento en el manejo de situaciones de salud en el campo escolar.

En Colombia, existen datos que ratifican la existencia de problemas de TDAH con impacto en el campo educativo, lo cual dificulta la inclusión de dichos estudiantes en los procesos de enseñanza, aunado a que los docentes no cuentan con bases sólidas en el tratamiento pedagógico de estudiantes con este trastorno. Como lo mencionan Miranda, Barrios, Duque, Burgos \& Salazar (2018) la prevalencia de TDAH en Colombia es de $5,29 \%$, y en regiones como Sabaneta, es del 20,4\%. Esta problemática por lo general comienza en la etapa escolar, lo que hace que este periodo sea trascendental la caracterización de niños con este trastorno; sin embargo, para los referidos autores, las competencias de los docentes para la detección de este trastorno no son las adecuadas. Ello genera un vacío pedagógico, puesto que se necesitan procesos de actualización y formación docente y equipos interdisciplinarios, que permitan una intervención integral 
en el campo pedagógico y convivencial, logrando atender las dificultades como el déficit de atención.

En las dificultades del aprendizaje que presentan los estudiantes en el ámbito escolar, es importante utilizar la programación neurolingüística ligada a estilos de aprendizaje en el campo educativo para potenciar la atención voluntaria en estudiantes. La programación neurolingüística, (PNL) se especifica como el conjunto de actitudes, herramientas, percepciones y aprendizajes que son capaces de hacer cambiar la forma de pensar y de actuar (Torres, Díaz \& Pérez, 2012). Por medio de estrategias, lo que se pretende es desarrollar las potencialidades de los estudiantes de transición y generar aprendizajes tendientes al mejoramiento continuo en el ámbito atencional logrando resultados efectivos en el ámbito académico, humano y social.

En este sentido Gamboa, Briceño \& Camacho (2015) en cuanto a los estilos de aprendizajes, precisan que los estilos de aprendizaje están estrechamente en concordancia con los canales sensoriales. Por lo que se plantea que los seres humanos conciben tiendo como referente tres canales de percepción: visual, auditivo y kinestésico, que es conocido como modelo VAK. Es necesario identificar el estilo de aprendizaje predominante en cada estudiante para focalizar mejor la programación neurolingüística y poder potenciar la atención voluntaria en los estudiantes.

Al respecto de la atención voluntaria, Hernández (2012) indica que la teoría histórico-cultural refiere que la atención voluntaria comprende el producto de un complejo desarrollo socio-histórico, vinculado al desarrollo del niño y a la adquisición del lenguaje. A partir de ello se puede precisar que el infante, va adquiriendo y fortaleciendo los canales sensoriales y los procesos psicológicos básicos, como la atención, memoria, percepción y el lenguaje con los cuales fortalece la focalización de la atención, puesto que permite que se adhiera mayor información a los procesos del pensamiento.

La Fundación INECO (2019), indica que la Organización Mundial de la Salud más del 4\% de la población mundial tiene TDAH, situación que provoca en la escolaridad un riesgo eminente ya que los docentes no están formados para educar estudiantes con TDAH. Así Miranda et al. (2018) plantea la existencia de TDAH en Colombia es de 5,29\%, y en regiones como Sabaneta, alcanza el 20,4\%. Este trastorno se hace más evidente en la etapa es escolar. Al respecto, Castaño et al. (2018) en un estudio realizado 
en la ciudad de Buenaventura, precisan la prevalencia por déficit de atención determinándolo como un problema local. De esta forma el contexto escolar se vuelve foco hostil e inclusivo, para estudiantes con NEE (necesidades educativas especiales) o NEET (necesidades educativas especiales transitorias).

En la ruta seguida en la investigación, se plantea el estudio de la programación neurolingüística (PNL) Torres, Díaz \& Pérez (2012) como una serie de actitudes, herramientas de aprendizajes que logran modificar la estructura del pensamiento y proceder de las personas. Según lo anterior por medio de diferentes estrategias, lo que se pretende es desarrollar las potencialidades de los estudiantes y generar aprendizajes tendientes al mejoramiento continuo en el ámbito atencional logrando resultados efectivos en el ámbito académico, humano y social.

"La Programación Neurolingüística es una adecuada herramienta que permite abordar la enseñanza de las habilidades propias de la educación para la paz, en general, y de la mediación escolar en particular" (Ramos, 2003, p. 17). De esta forma el PNL es visto desde diferentes panoramas de la vida social y cotidiana como una herramienta de solución educativa que permite perfeccionar el currículo para poder dar una mejora significativa al proceso de enseñanza- aprendizaje. Huerta (2005) logra definir tres características de la PNL:

(i) Programación: Consiste en diseñar la estructura de un sistema para lograr los resultados esperados. (ii) Neuro: personifica la idea de que todo comportamiento se derivan de los procesos neurológicos. Y (iii) Lingüística: el lenguaje y la comunicación comienza con sistemas nerviosos están representados y organizados secuencialmente.

Para Bavister \& Vickers (2014) la PNL es una forma de comunicación donde predomina el uso del pensamiento que modifican de forma positiva el comportamiento de los seres humanos. Ramírez (2018), trata la PNL como instrumento positivo e importante en cuanto facilita la enseñanza del maestro y el aprendizaje del estudiante. De lo cual, se pude inferir que la neurolingüística, trata en sí de un programa que activa los procesos neurológicos y los modula a través del lenguaje, teniendo en cuenta las dinámicas dialógicas, la cuales permiten la construcción relacional y del conocimiento así; como se utiliza la atención voluntaria, como proceso básico a nivel neurológico, con el fin de focalizar estrategias de aprendizaje tendientes al mejoramiento del aprendizaje de los estudiantes de forma permanente, en este orden resaltar el estilo de aprendizaje sobresaliente en cada estudiante para precisar las estrategias didácticas en las aulas de clase. 


\section{Enfoque teórico de la programación neurolingüística}

La teoría de la comunicación, es la teoría fundamental en la programación neurolingüística porque es a partir de la comunicación o lenguaje que se puede generar cambios positivos en los procesos neurológicos que precisan el comportamiento de las personas dentro de una sociedad determinada mejorando sus relaciones interpersonales. Para Sánchez \& Campos (2009) las teorías de la comunicación esbozan el fenómeno de la comunicación como una acción importante que el individuo realiza diariamente, en este aspecto es el sujeto que escoge como comunicarse de forma precisa que los demás lo puedan comprender lo que está expresando. Por otra parte Jaruffe \& Pomares (2011) plantean que la programación neurolingüística, se rige desde el constructivismo explicando la realidad como una iniciativa y no como un descubrimiento. Además manifiestan que se trata de maneras personales de relacionarse, que se explica desde las ciencias cognitivas.

Desde el constructivismo, se sostiene la postura de que los seres humanos no construyen su mundo solo, sino teniendo como referencia el mundo exterior ya que la comprensión del mundo real está dada en el contexto. Por lo que cabe la posibilidad que a través de la programación neurolingüística (PNL) ajustar la actitud y aptitud en el individuo. Para la ciencia cognitiva, el propósito es exponer cómo se piensa, y a partir del constructivismo, como se edifican las formas de pensamiento. Según lo anterior, es transcendental que los educandos obtengan herramientas que les permita aprender a aprender, en este sentido la PNL puede contribuir a generar mejoras en el pensamientos, potencializar habilidades y conductas con estrategias didácticas según objetivos de aprendizaje y niveles de desempeño del nivel educativo.

\section{Estilos de aprendizaje}

Comprende las estrategias preferidas para Alanís \& Gutiérrez (2011) que permite: compilar, interpretar, estructurar y pensar sobre la nueva información. Por lo tanto, este tipo de estrategias deben estar implementadas en el campo educativo, ya que los maestros deben adecuar la didáctica, pedagogía y practica pedagógica centrada en reorganizar y resignificar el currículo tradicional, con el fin de flexibilizar e innovar a través de estrategias como PNL en la primera información logrando la apropiación de los procesos neurológicos focalizados y direccionados para mejorar el aprendizaje.

En este caso, Romo, López \& López (2006) plantean que según el modelo de la PNL sobre los estilos de aprendizaje, se clasifica a los estudiantes en: visuales, auditivos 
y kinestésicos. En concordancia, a los diseños de los procesos enseñanza-aprendizaje deben ir direccionados a la canalización de los canales de percepción visual, auditivo y kinestésico, que son el hilo conductor para que los estudiantes identifiquen y pondere diversos estilos de aprendizaje. Así, Gamboa, Briceño \& Camacho (2015) plantean que una programación de enseñanza debe estar organizada en lograr que el alumno desarrolle sus diferentes canales de percepción para que este sea autodidacta en su forma de aprender. Es significativo que el docente conozca los canales de percepción sensorial predominantes de sus estudiantes y que ellos sean consiente de estos para que se puedan utilizar con mayor frecuencia y se potencialicen. A continuación se plantean sugerencias a tener en cuenta en el trabajo de aula según Fleming \& Mills (2007) en relación a cada estilo de aprendizaje VARK.

(i) Estrategias de enseñanza para el estilo Visual: este tipo de estudiantes aprenden más fácilmente en forma escrita, con apoyo de los mapas conceptuales, los diagramas, así como los modelos, los cuadros sinópticos, $\mathrm{y}$ animaciones computacionales, tales como videos, fotografías e ilustraciones.

(ii) Por lo contrario los estudiantes Auditivos: aprenden con mayor facilidad con estrategias de enseñanza verbales, es decir repetir sonidos parecidos, así como escuchando, participando en debates, discusiones y las confrontaciones, el desarrollar las lluvias de ideas, así como leer el mismo texto con distinta reflexión, la lectura guiada y comentada.

(iii) Para los estudiantes Quinésico o kinestésico los recursos didácticos deben ser: el juego de roles y las dramatizaciones, las dinámicas grupales que requieran sentarse y pararse, el uso del pizarrón para resolver problemas, manipulación de objetos para hacer posible la explicación de fenómenos, y los gestos para acompañar las instrucciones orales.

Los estilos de aprendizaje y los canales sensoriales fueron estudiados por Gamboa, Briceño \& Camacho (2015) ellos afirman que los estilos de aprendizaje están sujetos a los canales sensoriales. Se plantea que los seres humanos pueden percibir el mundo desde tres canales de percepción como: el visual, el auditivo y el kinestésico, lo cual se conoce como modelo VAK. Así se adopta una posición constructivista en el sentido de que las personas actúan de acuerdo con la forma en que entienden y representan el mundo. Por 
tanto, juega un rol importante en los canales sensoriales en el aprendizaje, destacandose una predominancia de los procesos básicos neurológicos, como la percepción, y a través de dichos procesos es necesario encausar el plan de aula, para que no exista fracaso escolar, sino una aprehensión del conocimiento.

\section{Atención voluntaria}

Para Hernández (2012) sin la atención sería imposible comprender el mundo que nos rodea, por lo tanto no podría estructurarse con un orden lógico. La forma en que los factores emocionales se combinan con los procesos de memoria y atención para producir un desempeño calificado siendo fundamental para la comprensión de la cognición humana.

Sin embargo, es solo recientemente que la interacción de las emociones y el control cognitivo ha recibido mucha atención en la investigación del desempeño humano. Así, se puede destacar lo que mencionamos anteriormente, la atención, no solo se involucra a los sentidos, sino a otros procesos básicos como la percepción, la memoria, para poder llevar a cabo y emprender el proceso atencional, donde se selecciona, se filtra la información, con el fin de lograr una aprehensión del conocimiento y un verdadero aprendizaje. Es por ello que la atención, tiene una relación con la aplicación voluntaria con los sentidos y los procesos cognitivos, enfocando especial cuidado en lo asimilado para lograr cumplir con la tarea propuesta.

La atención voluntaria para Hernández (2012) es aquella que ocurre cuando un estímulo, por su potencia capta la atención sujeto. Por lo tanto no depende del ser humano, en otras palabras es propia del individuo, y todas las personas la tenemos. En muchas situaciones de la vida de las personas la atención esta estimulada por eventos externos dentro de un determinado contexto o situación. En este orden de ideas el comportamiento de un niño muy pequeño, no estamos en condiciones de descubrir actos de comportamiento voluntario en plenitud. Es solo en una etapa avanzada del desarrollo psicológico individual que la atención voluntaria comienza a adquirir esa importancia central y sólida.

\section{Problemas en torno a la atención en la educación}

Los problemas de atención se agrupan en el Trastorno de Atención e Hiperactividad (TDAH). Norton (2013) plantea que el TDAH “(...) es un trastorno del neurodesarrollo caracterizado por la dificultad para prestar atención, actividad excesiva e 
impulsividad" (p.2). En el ámbito de clínico, definen al TDAH como el determinado grado de déficit de atención y/o hiperactividad que denota marcada impulsividad y que resulta desadaptativo e incoherente en relación al nivel de desarrollo del niño, y que está presente antes de los 12 años de edad (Hidalgo \& Sánchez, 2014). El TDAH es un esquema constante de desatención y/o hiperactividad-impulsividad, que es mucho más fuerte y grave superior a lo que constantemente percibimos en la cotidianidad en dificultades similares (Vélez \& Vidarte, 2012). El trastorno por déficit atencional con hiperactividad es un problema del desarrollo del ser humano determinado por niveles de inatención, que reflejan actitudes impulsivas inadecuadas. La presencia de los síntomas a menudo se identifica en la primera infancia (Begoña, 2015).

Analizando las precisiones de Alsina et al., (2014) a la persona con TDAH se le dificultad seguir instrucciones, dificultando el proceso de enseñanza-aprendizaje. Esta situación de desatención puede ocasionar mala convivencia en el aula, un aprendizaje más lento, matoneo, desmotivación, baja autoestima entre otro. Fernández \& Flórez (2016) plantean que la atención no es un asunto unitario por lo contrario es conjunto de diferentes elementos que actúan sincrónica. De esta forma influyen una serie de factores que pueden influir para obtener un aprendizaje significativo si las condiciones son óptimas en cuanto al desarrollo integral del estudiante.

Partiendo de la población objeto de estudio, es pertinente enfatizar las dimensiones que permiten la formación integral de estudiantes en edad preescolar que fue expuesto por el Ministerio de Educación Nacional en Colombia desde los Lineamientos Curriculares de Preescolar (la dimensión corporal, la dimensión cognitiva, la dimensión comunicativa, la dimensión estética y la dimensión ética) como precisiones fundamentales (MEN, 1996). En ella, se precisa que las dimensiones del docente deben sostener todos los procesos de formación de los estudiantes desde su contexto personal, familiar y social. Avanzando en el documento se encuentra, la metodología donde se describe el diseño metodológico, el tipo de investigación, el corpus de investigación, en este caso la investigación es exploratoria y explicativa, debido a que explicará cómo está ligado: la programación neurolingüística, atención voluntaria y estilos de aprendizaje con otras disciplinas, para proveer posibles recomendaciones y soluciones al problema desde el campo pedagógico.

\section{Materiales y métodos}


El estudio se enmarca en el método hipotético deductivo, que parte de la elaboración de las hipótesis según las variables y dimensiones para su respectiva correlación. Las hipótesis deben ser comparadas con los hechos para ser aceptadas o negadas. En este sentido, se pretende potenciar la atención voluntaria de estudiantes de grados transición aplicando una propuesta de programación neurolingüística ligada a estilos de aprendizaje. Hernández, Fernández \& Baptista (2010) precisa que de la teoría se derivan expresiones lógicas, utilizando el razonamiento deductivo, que se inicia con las hipótesis y que el investigador se propone a someter a prueba.

Se configura esta investigación desde una tipología aplicada con enfoque cuantitativo, El enfoque cuantitativo se encuentra el contraste de diversas teorías a partir de una serie de hipótesis planteadas, haciéndose eminente obtener una muestra, representativa de una población investigada. Por ello, la recopilación de dato se hizo por medio de análisis estadístico teniendo en cuenta la población objeto de estudio. El nivel de este estudio, es el explicativo según Hernández -Mendoza (2018) su interés consiste en explicar porque suceden los fenómeno y como se muestra, también se trata de explicar porque se relaciona con dos o más variables. Desde el nivel explicativo se profundiza en las variables: programación neurolingüística, estilos de aprendizaje y atención voluntaria, para determinar como la variable independiente modifica la variable dependiente y proponer posibles soluciones.

El diseño adoptado es experimental, que se enmarca en el sub diseño cuasi experimental, que responde a una pre prueba y otra pos prueba con grupos intactos un grupo experimental (GE) y un grupo control (GC). Se determinó una población de 2634 personas (94 docentes y 2540 estudiantes). Según Hernández, Fernández \& Baptista precisan la población como, "el grupo de todos los elementos que reúnen determinados requisitos" (2010, p. 174). La muestra está conformada por 425 estudiantes del grado transición, la cual la conforma 4 colegios oficiales de la ciudad de Buenaventura Colombia, ubicados en la zona urbana con niños de 5 años de edad, esta involucran a 15 docentes de los grados anteriormente mencionados. El muestreo es probabilístico en cuanto se aplican parámetros estadísticos para la selección de la muestra. Según el resultado como mínimo se deben elegir 334 estudiantes, pero en este estudio se escogió una muestra de 425 estudiantes.

Fórmula de tamaño de muestra: 


$$
n=\frac{1.96^{2} * 0.5 *(1-0.5) * 2540}{0.05^{2}(2540-1)+1.96^{2} * 0.5 *(1-0.5)}=333.8 \cong 334
$$

La técnica utilizada en esta investigación fue encuesta de opinión y test. Al respecto Flores (2015) plantea que las técnicas, son los procedimientos o manera de proceder del investigador para reunir información, es decir permite los datos que se necesita para lograr los objetivos. Para el desarrollo de los objetivos diseñados en este trabajo se utilizaron fuentes de información primaria utilizando como instrumento cuestionario y hoja de evaluación. En cuanto a la validez de los instrumentos Hernández, Fernández \& Baptista (2010) plantean que la validez, muestra en un instrumento lo que este debe medir real mente en una variable. En este orden de ideas la validación de los instrumentos fue admitida, revisada y aprobada por 7 expertos, la congruencia de jueces expertos se realizó mediante el Coeficiente de validación V de Aiken. Los dos instrumentos son válidos (Test de PNL infantil ligado a los estilos de aprendizaje, 0.95 en pertinencia, 0.91 en relevancia, 0.94 en claridad y 1 en suficiencia; encuesta a docentes, 0.95 en pertinencia, 0.98 en relevancia, 0.93 en claridad y 01 en suficiencia).

La confiabilidad del test de programación neurolingüística aplicado a los estudiantes dio un índice de 0,8239 y la encuesta de opinión aplicada a docentes muestra una confiabilidad de 0,8126 ; mostrando que los instrumentos son fiables y pueden ser aplicados al resto de la muestra utilizando el método de Kuder-Richarson 20. En cuanto a los criterios de inclusión se encuentran, (i) Colegios de zona urbana BuenaventuraColombia, (ii) Docentes del nivel preescolar, (iii) 4 Colegios oficiales (Instituciones Educativas: Teófilo Roberto Potes, Termarit, Normal Superior y José María Cabal), (iiii) Estudiantes de grado transición de colegios mencionados. Entre los criterios de exclusión se plantean, (i) Colegios oficiales de la zona rural, (ii) Docentes de otros niveles de escolaridad, (iii) Estudiantes de básica y media. La investigación fue aprobada por el Comité de ética de la institución, presentando integridad científica la confidencialidad de los participantes y del material obtenido, la exigencia del consentimiento informado, turnitin, normas APA, la validación de los instrumentos para la recolección de datos realizada por jueces expertos, de acuerdo al rigor científico y el cumplimiento del código de ética de la Universidad.

\section{Resultados}

Según la prueba Kolmogorov-Smirnov para normalidad, y la prueba U de MannWhitney para el contraste de hipótesis; se aplicó la encuesta de opinión a los docentes se 
determinó la viabilidad de la investigación en cuanto determinó que los docentes cuentan con estudiantes con déficit de atención en sus aulas de clase, no se encuentran formados para asumir dicho rol y las docentes consideran que una propuesta de programación neurolingüística infantil ligada a los estilos de aprendizaje contribuye a potenciar la atención voluntaria de los estudiantes en los espacios académicos (ver Tabla 1 y figura 1).

Tabla 1

Estilos de aprendizaje, Instituciones Educativas focalizadas

\begin{tabular}{lcccccc}
\hline \multicolumn{7}{c}{ Instituciones Educativas } \\
& IE 1 & IE 2 & IE 3 & IE 4 & Totales & $\%$ \\
Auditiva & 36 & 56 & 17 & 27 & 136 & $32 \%$ \\
Kinestésico & 42 & 67 & 19 & 43 & 171 & $40 \%$ \\
Visual & 45 & 25 & 16 & 31 & 117 & $28 \%$ \\
& & & & 424 & $100 \%$ \\
\hline
\end{tabular}

Fuente: Elaboración Propia

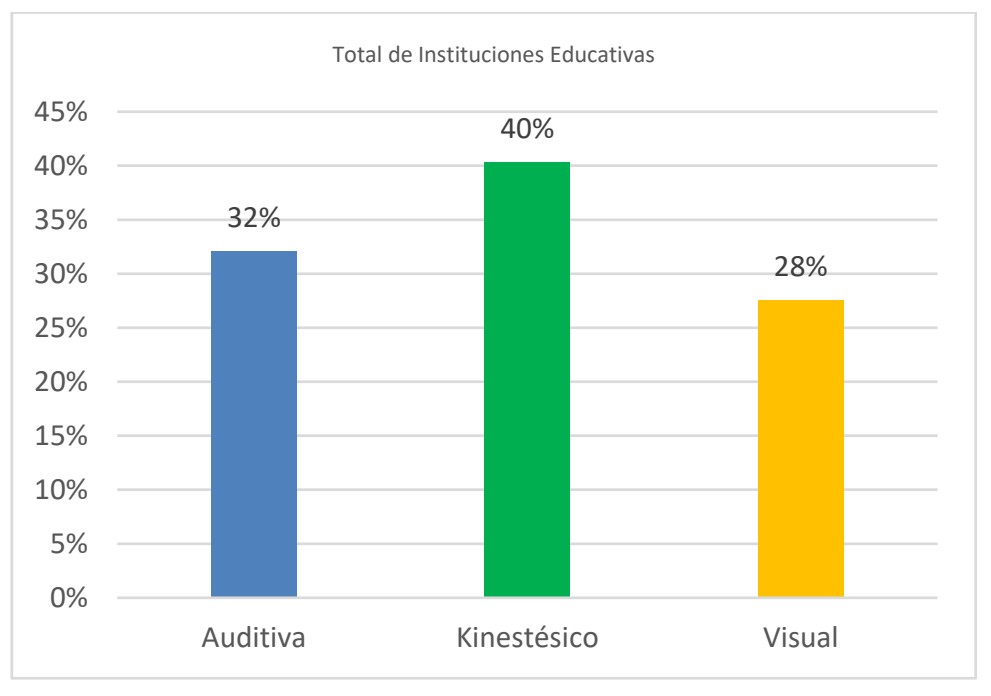

Figura 1. Estilos de aprendizaje, por Instituciones Educativas focalizadas Fuente: Elaboración Propia

Tabla 2

Resultados de los Test aplicados a los Estudiantes

\begin{tabular}{|c|c|c|c|c|c|c|c|c|}
\hline \multicolumn{3}{|c|}{ Longitudinal } & $\begin{array}{l}\text { Dimensión } \\
\text { cognitiva }\end{array}$ & $\begin{array}{l}\text { Dimensión } \\
\text { estética }\end{array}$ & $\begin{array}{l}\text { Dimensión } \\
\text { comunicativa }\end{array}$ & $\begin{array}{l}\text { Dimensión } \\
\text { ética }\end{array}$ & $\begin{array}{l}\text { Dimensión } \\
\text { corporal }\end{array}$ & Evaluaciones \\
\hline \multirow[t]{2}{*}{ Pretest } & $\mathrm{N}$ & Válido & 426 & 426 & 426 & 426 & 426 & 426 \\
\hline & & Perdidos & 0 & 0 & 0 & 0 & 0 & 0 \\
\hline
\end{tabular}




\begin{tabular}{|c|c|c|c|c|c|c|c|c|}
\hline & \multicolumn{2}{|l|}{ Media } & 2,36 & 2,03 & 3,16 & 1,46 & 2,60 & 11,61 \\
\hline & \multicolumn{2}{|c|}{ Error estándar de la media } &, 041 &, 041 &, 046 & 029 &, 038 &, 145 \\
\hline & \multicolumn{2}{|c|}{ Desviación estándar } & ,854 &, 844 & ,945 & ,606 & ,777 & 3,003 \\
\hline & \multicolumn{2}{|l|}{ Rango } & 3 & 3 & 4 & 2 & 3 & 15 \\
\hline & \multicolumn{2}{|l|}{ Mínimo } & 0 & 0 & 0 & 0 & 0 & 0 \\
\hline & \multicolumn{2}{|l|}{ Máximo } & 3 & 3 & 4 & 2 & 3 & 15 \\
\hline & \multirow[t]{3}{*}{ Percentiles } & 25 & 2,00 & 1,75 & 3,00 & 1,00 & 2,00 & 10,00 \\
\hline & & 50 & 3,00 & 2,00 & 3,00 & 2,00 & 3,00 & 13,00 \\
\hline & & 75 & 3,00 & 3,00 & 4,00 & 2,00 & 3,00 & 14,00 \\
\hline \multirow[t]{11}{*}{ Postest } & \multirow[t]{2}{*}{$\mathrm{N}$} & Válido & 426 & 426 & 426 & 426 & 426 & 426 \\
\hline & & Perdidos & 0 & 0 & 0 & 0 & 0 & 0 \\
\hline & \multicolumn{2}{|l|}{ Media } & 2,42 & 2,40 & 3,70 & 1,85 & 2,60 & 12,96 \\
\hline & \multicolumn{2}{|c|}{ Error estándar de la media } & 040 & 039 & 029 & 018 & 038 &, 118 \\
\hline & \multicolumn{2}{|c|}{ Desviación estándar } & 828 & ,797 &, 590 &, 371 & ,777 & 2,433 \\
\hline & \multicolumn{2}{|c|}{ Rango } & 3 & 3 & 3 & 2 & 3 & 13 \\
\hline & \multicolumn{2}{|l|}{ Mínimo } & 0 & 0 & 1 & 0 & 0 & 2 \\
\hline & \multicolumn{2}{|l|}{ Máximo } & 3 & 3 & 4 & 2 & 3 & 15 \\
\hline & \multirow[t]{3}{*}{ Percentiles } & 25 & 2,00 & 2,00 & 3,75 & 2,00 & 2,00 & 11,75 \\
\hline & & 50 & 3,00 & 3,00 & 4,00 & 2,00 & 3,00 & 14,00 \\
\hline & & 75 & 3,00 & 3,00 & 4,00 & 2,00 & 3,00 & 15,00 \\
\hline
\end{tabular}

Fuente: Elaboración propia

En líneas generales se puede apreciar un aumento en el valor de las evaluaciones comparando el inicio con el final de ciclo, es decir comparando las evaluaciones pre-test y pos-test (estadísticos de la media y la mediana).

Tabla 3

Análisis de la Prueba de U Mann-Whitney

Estadísticos de prueba ${ }^{\mathrm{a}}$

U de Mann-Whitney

W de Wilcoxon

$\mathrm{Z}$

Sig. asintótica (bilateral)

Evaluaciones

$$
\begin{array}{r}
17219,000 \\
32619,000 \\
-3,911 \\
, 000
\end{array}
$$

a. Variable de agrupación: Grupos cuasiexperimentales

De acuerdo a la información muestral como $p$-valor $=0,000<\alpha=0.05$; se rechaza $\mathrm{H}_{0}$ con un nivel de significancia de $5 \%$ se rechaza la hipótesis nula, es decir, la programación neurolingüística ligada a estilos de aprendizajes influye en la atención voluntaria en estudiantes de transición en Buenaventura, Colombia durante el año 2019.

\section{Discusión}


Los resultados definieron que la propuesta de programación neurolingüística ligada a estilos de aprendizajes influye positivamente en la potencialización de la atención voluntaria en estudiantes de transición en Buenaventura-Colombia durante el año 2019. De esta forma al confrontar el grupo control con el grupo experimental, en el post test se logra un valor de p de 0.000 , precisando diferencias significativas al ser p-valor $<0.05$, en el post test de los grupos control y el grupo experimental, dicho resultado está en consonancia en lo hallado por Velezvía (2020) se aplicaron dos instrumentos: uno de Programación Neurolingüística para determinar el canal de aprendizaje de preferencia de los estudiantes y un test de aprendizaje significativo como resultado se encontró que el canal principal de aprendizaje es el auditivo, seguido del visual y por último el kinestésico. Con correlaciones por encima del 0,05 los resultados se diferencian en que para la actual investigación canal principal es el kinestésico, seguido del auditivo y por último el visual.

Asimismo, el trabajo de investigación de Vitela (2018) comprende un estudio mixto, en el que se aplicó el modelo “investigación-acción-participante," con 120 estudiantes de Licenciatura en Preescolar y a quienes se les aplicó un test para evaluar sus estilos de aprendizaje. Esto tuvo como base un Modelo de Programación Neurolingüística, conformado por 40 ítems de opción múltiple, el cual evalúa los estilos visual, auditivo y kinestésico. En ello, los estudiantes potenciaron su atención y reconocieron el estilo de aprendizaje predominante e identificaran otros. Esta investigación es relevante porque procura identificar otros estilos de aprendizaje diferente a lo ya identificado, lo que hace pensar que es importante que los estudiantes potencien varios estilos de aprendizaje que permitan captar su atención, a fin de obtener mejores y variadas opciones para canalizar su aprendizaje.

Por su parte, Miranda, et al. (2018) describen los conocimientos en los docentes de básica primaria frente al trastorno por déficit de atención e hiperactividad (TDAH), el estudio arrojo que menos del $50 \%$ de los docentes no tienen conocimiento adecuado sobre el trastorno de déficit de atención e hiperactividad (TDAH). Lo pone en evidencia que existe la necesidad de plantear y ejecutar intervenciones sobre este tema en particular, para que se mejoren los procesos de enseñanza y aprendizaje en los niños y niñas que tienen problemas de atención voluntaria. En la presente investigación el 100\% de los docentes manifiestan no estar capacitados para atender estudiantes con déficit de atención; en este mismo sentido, todos ellos manifestaron tener estudiantes con dificultad en prestar atención o concentrarse de forma reiterativa. En todo este proceso, queda hacer 
una pregunta, que motivaría un estudio adicional: ¿Cómo están enfrentando estos docentes el problema de sus estudiantes con déficit de atención de forma idónea?

\section{Conclusiones}

Teniendo presente los resultados se cumple el objetivo general de la investigación estableciendo que la propuesta de programación neurolingüística ligada a estilos de aprendizajes tiene una influencia positiva para potencializar la atención voluntaria en estudiantes de transición en Buenaventura, Colombia durante el año 2019. Por ello, al comparar el grupo control y el grupo experimental en el post test se obtuvo un valor de $p$ de 0.000 , observándose, que si existen diferencias altamente significativas al ser p-valor $<0.05$, en el post test en los grupos control y experimental: comprobándose que la propuesta de programación neurolingüística ligada a estilos de aprendizajes tiene una influencia positiva para potencializar la atención voluntaria, y que a su vez casi todas las dimensiones tuvieron influencia positiva, excepto en la primera dimensión.

Por lo tanto, al comparar el grupo control y el grupo experimental en el post test se observó diferencias altamente significativas, siendo el $p$-valor $>0.05$, en el post test de los grupos control así como del grupo experimental: evidenciándose que la aplicación de la propuesta de programación neurolingüística ligada a estilos de aprendizajes no tiene una influencia positiva para potencializar la dimensión cognitiva.

\section{Referencias}

Alanis, P. \& Gutiérrez D. A. (2011). Los estilos de aprendizaje en estudiantes de telesecundaria. Visión Educativa IUNAES, Vol. 5, No 12, pp. 22-32. http://iunaes.mx/inicio/wp-content/uploads/2013/04/12-Marzo-de-2012.pdf

Alsina-M, G,; Amador C. JA.; Arroyo R, A., Badia G, A., Mar B, M; Contreras C, C., Mas S, B,. Mena P, B., Salat F, L., \& Saumell M. C.; (2014) Déficits de atención y trastornos de conducta. Editorial UOC, Barcelona. https://online.ucv.es/resolucion/deficits-de-atencion-y-trastornos-de-conducta/

Begoña, R. B. (2015). Detección e intervención del TDHA en educaciónal. Tesis de Maestría. Universidad Internacional de la Rioja. https://reunir.unir.net/bitstream/handle/123456789/2852/Bego\%C3\%B1a_Ripol _Baixas.pdf?sequence $=1 \&$ isAllowed $=\mathrm{y}$

Batlle V, S. \& Tomàs, J. (s.f). Disfunción de la Atención en la Infancia y la Adolescencia: Evaluación Intervención. 
http://www.centrelondres94.com/files/disfuncion_de_la_atencion_en_la_infanci a_y_la_adolescencia_evaluacion_e_intervencion.pdf

Bavister, S. \& Vickers, A. (2014). Programación Neurolingüística. Barcelona: Amat Castaño P. E. A., Sierra H. G. M., Sánchez A.D., Semenova M, N., Zapata B. N.,Salazar, C., y otros. (2018). Salud mental en víctimas de desplazamiento forzado por la violencia en Colombia. Editorial CES

Fernández, R. \& Flórez, J. (2016). La atención: bases fundamentales. Fundación Iberoamericana Down 21. Recuperado de: https://bit.ly/2yvzTMP

Fleming. N \& Mills. C (2007). Modelo VARK: sistemas de representación. http://www.cca.org.mx/profesores/cursos/cep21/modulo_2/modelo_vark.htm

Flores A. J (2015) Como hacer y evaluar una tesis. Editorial de la Universidad José Carlos Mariátegui. Moquegua, Perú.

Fundación INECO (2019). https://www.fundacionineco.org/mas-del-4-de-la-poblacionmundial-tiene-tdah/

Gamboa M. M. C., Briceño M. J. J., \& Camacho G. J. P. (2015). Caracterización de estilos de aprendizaje y canales de percepción de estudiantes Universitarios Opción, vol. 31, núm. $\quad 3, \quad 2015, \quad$ pp. 509-527. https://www.redalyc.org/pdf/310/31045567026.pdf

Hernández G. A. I., (2012). Procesos psicológicos básicos. México: Red tercer milenio. (1 ra Ed). México. http://www.aliatuniversidades.com.mx/rtm/?wc$\underline{\text { api }=\text { wcxp_quick view\&product }=2804 \& \text { ajax }=\text { true }}$

Hernández-Sampieri R. \& Mendoza T. C. (2018) Metodología de la investigación. (1ra ed.). México, D.F., México: McGraw- Will Interamericana.

Hernández, R., Fernández, C. \& Baptista, P. (2010). Metodología de la investigación. (5a ed.) México, D.F., México: McGraw-Hill Interamericana.

Hidalgo V. M., \& Sánchez S. L. (2014). Trastorno por déficit de atención e hiperactividad (TDAH). Pediatra Integral 2014; XVIII (9): 609-623. https://www.pediatriaintegral.es/wp-content/uploads/2014/xviii09/03/n9-609623_Ines\%20Hidalgo.pdf

Huerta, S. (2005). Aprendizaje Estratégico Como Enseñar a Aprender y Pensar Estratégicamente. Lima- Perú: San Marcos. 
Jaruffe, A. \& Pomares, M. (2011). Programación neurolingüística. ¿Realidad o mito en Psicología y Ciencias Cognitivas?. Revista Duazary, 2(1), 243-250. https://bit.ly/3gmHePF

Ministerio de Educación Nacional (1996). Serie lineamientos curriculares preescolares. Bogotá: MEN. $\quad$ https://www.mineducacion.gov.co/1759/articles339975 recurso 11.pdf

Miranda, A., Barrios, D., Duque, L., Burgos, E. \& Salazar, J. (2018). Conocimientos sobre TDAH de los docentes de primaria de colegios públicos de Sabaneta, Antioquia. Revista Colombiana de Psiquiatría, 47(3), 165-169. http://www.scielo.org.co/pdf/rcp/v47n3/0034-7450-rcp-47-03-00165

Norton, J. (2013). TDAH Guía para padres sobre medicamentos. The American Academy of Child and Adolescent Psychiatry, 1-73. https://www.psychiatry.org/File\%20Library/Psychiatrists/Practice/ProfessionalTopics/Child-Adolescent-Psychiatry/adhd-parents-medication-guide-spanish.pdf

Ramírez, J. (2018). El uso de la PNL como herramienta en el proceso de enseñanza aprendizaje de la ere aplicada a los estudiantes del grado noveno del Colegio Integrado Camacho Carreño del Municipio de Suratá. [Tesis de Grado]. Bucaramanga: Universidad Santo Tomás. Recuperado de: https://bit.ly/3gjTaln

Ramos, C. (2003). Un mirar, un decir, un sentir en la Mediación Educativa. Buenos Aires: Librería Histórica.

Romo A, M. E., López R, D., \& López B, I. (2006). ¿Eres visual, auditivo o kinestésico? Estilos de aprendizaje desde el modelo de la Programación Neurolingüística (PNL). Revista Iberoamericana De Educación, 38(2), 1-10. https://doi.org/10.35362/rie3822664

Sánchez, L. y Campos, M. (2009). La Teoría de la comunicación: diversidad teórica y fundamentación epistemológica. Revista Académica de la Federación Latinoamericana de Facultades de Comunicación Social. https://dialnet.unirioja.es/descarga/articulo/3719687.pdf

Torres, S. J. L., Díaz F.J.T. \& Pérez C. E., (2012). Programación Neurolingüistica: herramienta comunicacional efectiva de un liderazgo proactivo. Revista Electrónica de Psicología en Iztacala. Vol.15 No3 https://www.iztacala.unam.mx/carreras/psicologia/psiclin/vol15num3/Vol15No3 Art10.pdf 
Vélez Á, C., \& Vidarte, J. (2012). Trastorno por déficit de atención e hiperactividad (TDAH), una problemática a abordar en la política pública de primera infancia en Colombia. Revista de Salud Pública. 14, 113-128. DOI: https://doi.org/10.1590/S0124-00642012000800010

Velezvía, P. (2020) El sistema visual auditivo kinestésico y su relación con el nivel de aprendizaje de los estudiantes de la facultad de ciencias de la educación de la UNA-Puno-2019. [Tesis Doctoral]. http://repositorio.unap.edu.pe/handle/UNAP/13489

Vitela, V. (2018) Los estilos de aprendizaje múltiples en el nivel preescolar. Revista Iberoamericana de Producción Académica y Gestión Educativa. https://pag.org.mx/index.php/PAG/article/view/782/1127 